\title{
La planification des plantations par les structures de première mise en marché dans la filière fruits et légumes. Comparaison des cas de la salade et de la pêche dans le sud-est de la France
}

Mireille Navarrete $^{\mathrm{a} *}$, Sophie Tordjman ${ }^{\mathrm{b}}$, Alexia Rouby ${ }^{\mathrm{a}}$

${ }^{a}$ Inra, Unité

d'Ecodéveloppement, site Agroparc,

84914 Avignon Cedex 09, France

navarret@avignon.inra.fr

b Inra, SADAPT, RD10, 78026 Versailles Cedex France
${ }^{*}$ Correspondance et tirés à part

Reçu le 15 janvier 2003 Accepté le 30 avril 2003

Fruits, 2003, vol. 58, p. 261-274 (C) 2003 Cirad/EDP Sciences All rights reserved

DOI: $10.1051 /$ fruits:2003014

RESUMEN ESPAÑOL, p. 274

\begin{abstract}
Planning of planting by the commercial organizations of fruit and vegetables. Comparison of salad vegetable and peach cases in South-East France.
\end{abstract}

Abstract - Introduction. The commercial organizations try to plan the planting decisions of the growers in order to fit to the requirements of the distributors. The aim of the study was to analyze the collective planning of planting on two species (salad vegetable and peach), and show how it integrated agronomical constraints for each species and retail outlets of the commercial organizations. Methods. We conducted a survey with the technical advisors of nine commercial organizations located in the South-East of France and some of the growers who supply them. Results. All the organizations marketing salad vegetables influenced their suppliers on the choice of product (lettuce, batavia, etc.) and the planting dates. This enabled the spacing out of the harvest dates and control of the variability of the products at the collective level. There were various levels of coordination depending on the commercial firms and the requirements of the distributors. For peach, the marketing organizations tried to influence the fruit growers' choices on subspecies (peach/nectarine and white/yellow flesh) and variety. But, in fact, the only real effect observed was the reduction in the number of varieties planted, which controls the variability of products but not the harvest date. Discussion and conclusion. The study enabled the comparison of an annual (salad vegetable) and a perennial (peach) species according to four criteria: the interest in a collective planning of planting, its economical and agronomical efficiency and some difficulties growers are faced with to implement such collective decisions.

France / Provence Alpes Côte d'Azur / salads / peaches / farm planning / regional planning / phased planting / choice of species / marketing

La planification des plantations par les structures de première mise en marché dans la filière fruits et légumes. Comparaison des cas de la salade et de la pêche dans le sud-est de la France.

Résumé - Introduction. Les structures de première mise en marché tentent de planifier les plantations de leurs apporteurs pour faciliter l'adéquation, au moment des récoltes, entre l'offre en produits et les demandes de leurs clients. L'objectif de l'article est d'analyser les dispositifs de planification des plantations sur deux espèces, la salade et la pêche, et de montrer comment ils tiennent compte des contraintes agronomiques propres à chaque espèce et des débouchés des structures de mise en marché. Dispositif d'étude. Des enquêtes ont été réalisées auprès des techniciens de neuf structures de mise en marché du sud-est de la France, ainsi que d'agriculteurs approvisionnant ces structures. Résultats. Dans le cas de la salade, toutes les structures enquêtées influent sur le choix des sous-espèces et des dates de plantation. Cela leur permet de contrôler l'étalement des récoltes et la variabilité des lots de produits, mais avec des degrés variables suivant les structures et leurs débouchés. Dans le cas de la pêche, elles tentent d'influencer les arboriculteurs sur le choix des sous-espèces et des variétés, mais, concrètement, cela se traduit presque essentiellement par une réduction du nombre de variétés cultivées. Discussion et conclusion. L'étude permet de comparer la planification des plantations pour une espèce annuelle et une espèce pérenne : intérêt, degré d'efficacité tant économique qu'agronomique, difficultés rencontrées par les agriculteurs dans leurs exploitations.

France / Provence Alpes Côte d'Azur / salade / pêche (fruits) / planification de l'exploitation / planification régionale / plantation échelonnée / choix des espèces / commercialisation 


\section{Introduction}

Depuis de nombreuses années, les agriculteurs et les structures qui commercialisent leurs produits cherchent à coordonner leurs décisions avant ou en cours de campagne afin de répondre aux attentes des autres acteurs de la filière en terme de quantité et de qualité de produits. Dans le secteur des fruits et légumes, ces relations sont cruciales car il s'agit majoritairement de produits très périssables. Les agriculteurs cherchent à anticiper les besoins de la filière pour éviter de perdre des produits faute de débouchés immédiats. Pour les structures de première mise en marché, il s'agit de garantir le respect des engagements pris avec leurs clients et une régularité d'approvisionnement de leurs stations de conditionnement ; elles mettent donc en place des conseils et des cahiers des charges techniques pour inciter les agriculteurs à faire évoluer leurs systèmes. Cette tendance s'observe aussi bien dans les coopératives agricoles que chez des expéditeurs privés (cas de la salade étudié par Tordjman [1]) ou dans les industries de transformation (cas des légumes de conserve étudié par Capillon et Valceschini [2]). Il ne s'agit cependant pas d'intégration verticale avec internalisation de l'activité de production agricole par les structures de mise en marché. En effet, les agriculteurs restent maîtres de leurs moyens de production ; de plus, l'intervention des structures de mise en marché se limite aux techniques qui jouent le plus directement sur la qualité finale des produits. La réforme de l'Organisation commune des marchés fruits et légumes en 1996 a renforcé ces relations en encourageant la création d'organisations de producteurs $(\mathrm{OP})^{1}$.

\footnotetext{
${ }^{1}$ Une OP (organisation de producteurs) regroupe pour une durée de 5 ans un ensemble de producteurs et une ou plusieurs structures de première mise en marché conventionnées. Les producteurs s'engagent à apporter la totalité de leurs produits aux structures conventionnées. L'OP élabore un cahier des charges technique qui encourage une production de qualité et des pratiques respectueuses de l'environnement, et fournit un appui technique.
}

Le concept de coordination a été utilisé par les économistes pour désigner toutes les procédures que les différents acteurs mettent en place pour rendre compatibles leurs plans [3]. Pour ces derniers, la coordination est un moyen de garantir la qualité et de faire des économies sur les coûts de transaction. Capillon et Valceschini [2] ont mis en évidence plusieurs étapes dans la coordination entre une usine de transformation de légumes et ses apporteurs : sélection ex ante des agriculteurs, planification des semis ou plantations, suivis techniques des parcelles en cours de culture, ajustements en temps réel au moment de la récolte.

Cet article présente spécifiquement l'étape de planification des plantations, c'est-à-dire le moment où se font les choix d'espèces, de variétés, de dates et densités de plantation, ainsi que de porte-greffes et de formes fruitières pour l'arboriculture, des modes de culture (plein champ, abri plastique, serre) pour le maraîchage.

En maraîchage et en arboriculture, la planification des plantations est une étape essentielle pour maîtriser à la fois la distribution temporelle des quantités récoltées et l'hétérogénéité de qualité des produits [1, 4] qui sont deux objectifs importants des structures de mise en marché. En effet, l'étalement des récoltes permet de rentabiliser la station de conditionnement et de conserver la relation avec les clients tout au long de la période de production. D'autre part, quels que soient les critères de qualité recherchés, l'hétérogénéité des produits pose un problème dès lors que les centrales d'achat, qui commercialisent les $2 / 3$ des fruits et légumes, exigent des lots importants de produits de qualité homogène. Or les volumes produits par chaque agriculteur, à une date donnée et dans un créneau de qualité donné, sont généralement très faibles. L'allotement consiste donc à rassembler à la récolte des productions issues de parcelles et d'exploitations agricoles différentes, d'où l'intérêt d'anticiper ce processus par une coordination au moment des plantations.

Le premier objectif de nos travaux a été d'étudier comment la planification des 
plantations tenait compte des contraintes agronomiques propres à chaque espèce. À ce titre, nous avons comparé deux espèces, la pêche et la salade, pour lesquelles la date de récolte et les possibilités d'allotement sont pilotées de façon très différente d'un point de vue agronomique (encadré 1). En particulier, nous avons fait l'hypothèse qu'il est plus difficile de se coordonner sur une espèce pérenne (pêche) que sur une espèce annuelle (salade) car les choix techniques effectués à la plantation de la pêche engagent le producteur pour une dizaine d'années. De plus, les deux espèces étudiées présentent une certaine originalité par rapport aux travaux réalisés auparavant sur la gestion du bassin d'approvisionnement. Il s'agit de produits périssables, comme les légumes destinés à la transformation [2] : le délai recherché entre date de récolte et date de commercialisation est de 3 jours maximum pour la salade, 10 jours maximum pour la pêche, ce qui réduit considérablement la marge de manœuvre au moment de l'allotement puisque seuls les lots récoltés à peu près en même temps peuvent être regroupés. Au contraire, pour les céréales [9], les processus d'allotement peuvent être différés dans le temps grâce au stockage. D'autre part, il s'agit de produits non miscibles : la qualité d'une salade ou d'une pêche ne peut être modifiée par mélange avec un produit de qualité différente alors que cela est possible pour les céréales. De ce fait, la phase de culture (de la plantation à la récolte) est primordiale dans l'élaboration de la qualité finale des produits commercialisés, la phase post-récolte permettant seulement d'éviter une dégradation trop rapide de la qualité.

Par ailleurs, différents travaux comparant des espèces ayant des modes voisins d'élaboration de la qualité ont montré que les dispositifs de coordination varient entre structures de mise en marché suivant les débouchés ou les créneaux de qualité que celles-ci privilégient. Ainsi, dans le secteur des céréales, Le Bail [9] a montré que les entreprises de collecte de l'orge de brasserie cherchent à réduire la variabilité de la teneur en protéine des lots autour du taux optimal pour les brasseurs, tandis que, pour le blé meunier, elles cherchent à fractionner la production en lots ayant des taux

Les facteurs qui jouent sur la date de récolte varient entre les deux espèces salade et pêche. La date de récolte de la salade est essentiellement fonction de la date de semis qui, dans le sud de la France, peut s'étaler sur 5 mois [5]. La durée entre le semis et la récolte varie de $(50$ à 120$)$ jours selon la date du semis [6]. Pour une même date de semis, les variations de durée du cycle dépendent, par ordre d'importance décroissante, du mode de production (abri ou plein champ), du climat et, enfin, de la variété [5]. Les fluctuations climatiques peuvent introduire des ajustements dans la date de récolte de \pm 4 jours [7]. Dans le cas de la pêche, la date de récolte est essentiellement fonction de la variété ; chaque variété est récoltée pendant environ 10 jours et l'ensemble des variétés disponibles permet de produire pendant 4 mois [8]. Pour une même variété, les variations de dates de récolte sont d'origine climatique (d'un lieu à l'autre ou d'une année à l'autre)

Les possibilités d'allotement des produits dépendent de plusieurs critères. La distinction entre sous-espèces en salade (laitue, batavia, romaine, feuille de chêne, etc.) et en pêche (pêche jaune, pêche blanche, nectarine jaune, nectarine blanche) est obligatoire du fait de leurs différences visuelles importantes [6,8]. À l'intérieur d'une même sous-espèce, il existe une soixantaine de variétés de pêche et une dizaine de variétés de salade. La variété est un critère fréquent mais non systématique de distinction des lots. Par exemple, plusieurs variétés de pêches jaunes sont regroupées dans le même lot commercial si elles présentent les mêmes caractéristiques visuelles (forme, calibre, couleur de la peau). Cela est nécessaire du fait de l'abondance de variétés sur le marché. Enfin, les possibilités d'allotement dépendent de l'itinéraire technique, qui conditionne la qualité intrinsèque du produit.

de protéine différents et adaptés à différents débouchés (panification, biscuiterie).

Notre deuxième objectif a donc été d'analyser, pour chaque espèce, comment les structures de mise en marché adaptaient la planification des plantations à leurs débouchés.

\section{Dispositif d'étude}

Nous avons juxtaposé deux dispositifs d'enquête dans lesquels nous avons comparé plusieurs structures de mise en marché.

Le premier dispositif, sur salade, visait à analyser l'ensemble des procédures de coordination qui lient les structures de mise en marché et leurs apporteurs (dont fait partie la planification des plantations) et à identifier les difficultés rencontrées dans les exploitations du fait de la coordination [10].

Le second dispositif, sur pêche, visait à étudier comment les choix de plantation des arboriculteurs étaient influencés par les pépiniéristes et les structures de mise en marché [4].

Différents types d'acteurs ont donc été enquêtés : techniciens et commerciaux des structures de mise en marché, pépiniéristes, agriculteurs. Dans cet article, la description du dispositif de planification des plantations repose principalement sur les informations

\section{Encadré 1.}

Principaux déterminants de la date de récolte et des possibilités d'allotement de la salade et la pêche. 


\section{Navarrete et al.}

\section{Tableau I.}

Caractéristiques de neuf structures de mise en marché pour la salade ou pour la pêche, après enquêtes dans le bassin de production du sud-est de la France.

\begin{tabular}{|c|c|c|c|c|}
\hline $\begin{array}{l}\text { Espèce } \\
\text { étudiée }\end{array}$ & Structure & Volume commercialisé & Débouchés commerciaux ${ }^{1}$ & $\begin{array}{l}\text { Nombre d'apporteurs } \\
\text { pour l'espèce étudiée }\end{array}$ \\
\hline \multirow[t]{5}{*}{ Salade } & S1 & $4-5$ millions de pieds & $\begin{array}{c}\text { Frais, } \\
4^{\mathrm{e}} \text { gamme sous contrat }\end{array}$ & 40 dont 20 font $90 \%$ du volume \\
\hline & $\mathrm{S} 2$ & $10-12$ millions de pieds & Frais & $\begin{array}{l}60 \text { dont } 20 \text { gros producteurs } \\
2 \text { exploitations en propriété }\end{array}$ \\
\hline & S3 & 13 millions de pieds & $\begin{array}{c}\text { Frais, } 4^{\mathrm{e}} \text { gamme } \\
\text { (1 usine en propriété) }\end{array}$ & $\begin{array}{l}100 \text { dont } 20 \text { font } 80 \% \text { du volume } \\
1 \text { exploitation en propriété }\end{array}$ \\
\hline & S4 & 12 millions de pieds & Frais, $4^{e}$ gamme & 40 \\
\hline & S5 & 15 millions de pieds & Frais, produits de niche & 40 \\
\hline \multirow[t]{4}{*}{ Pêche } & P1 & $12000 t$ & $\mathrm{GMS}^{2}$, export, grossistes & 60 \\
\hline & P2 & $6000 t$ & GMS & 35 \\
\hline & P3 & $6000 t$ & GMS, export, grossistes & 27 \\
\hline & $\mathrm{P} 4$ & $13000 t$ & GMS, grossistes & 40 \\
\hline
\end{tabular}

données par les techniciens. En effet, ils sont un maillon essentiel de la coordination puisqu'ils servent d'interfaces entre les agriculteurs et les commerciaux. Nous avons donc cherché à décrire le travail de ces techniciens au moment de la planification des plantations: nature des relations qu'ils entretiennent avec les agriculteurs, type d'informations qu'ils recueillent et façon dont ils les traitent pour coordonner les plantations à l'échelle de la structure de mise en marché.

Lorsque les techniciens n'étaient pas en mesure d'exposer les débouchés commerciaux de la structure, les commerciaux ou les directeurs ont été également enquêtés.

Nos travaux n'ont pris en compte les enquêtes effectuées auprès des agriculteurs que pour préciser et éventuellement confronter leurs réponses avec le point de vue du technicien sur la planification des plantations.

Les différents acteurs n'ont été enquêtés qu'une seule fois, mais nous avons cherché à positionner leur discours dans une dynamique passée et future, à moyen terme.

Les enquêtes sur salade se sont déroulées dans le Roussillon, région du sud de la France qui regroupe $73 \%$ de la production de salade du Bassin Rhône-Méditerrannée $(\mathrm{BRM})^{2}$. Parmi les huit structures qui commercialisent $90 \%$ de la production de salade du Roussillon, nous en avons rencontré cinq bien représentatives du bassin par leurs débouchés commerciaux (tableau I). Pour la pêche, les enquêtes se sont déroulées auprès de quatre structures de mise en marché situées également en Roussillon (P1, P2 et P3) et Drôme (P4), qui sont les principales zones de production en France (tableau I). Si l'on ne peut prétendre à une bonne représentativité de ces

2 Le BRM est l'entité administrative pour l'OCM fruits et légumes qui regroupe les régions Provence-Alpes-Côte d'Azur, Languedoc-Roussillon et Rhône-Alpes ; c'est le principal bassin de production de la pêche et de la salade d'hiver. 


\begin{tabular}{|c|c|c|}
\hline Structure & $\begin{array}{l}\text { Exploitations concernées } \\
\text { par le dispositif }\end{array}$ & Dispositif \\
\hline S1 & Toutes les exploitations & $\begin{array}{l}\text { Influence sur le choix de la sous-espèce (en particulier jeunes pousses, } \\
\text { frisée très fine, chicorée sous abri) } \\
\text { Influence sur le choix des dates de plantations } \\
\text { (en particulier octobre à novembre et mars à mai) }\end{array}$ \\
\hline S2 & $\begin{array}{l}\text { Deux exploitations en propriété } \\
\text { et celles appartenant au GIE }\end{array}$ & Influence sur le choix de la sous-espèce et des dates de plantations \\
\hline S2 & Autres exploitations & $\begin{array}{l}\text { Information et ajustements à la marge pour le choix de la sous-espèce } \\
\text { et des dates de plantations }\end{array}$ \\
\hline S3 & Toutes les exploitations & Influence sur le choix des dates de plantations \\
\hline S4 & Toutes les exploitations & Influence sur le choix de la sous-espèce et des dates de plantations \\
\hline S5 & Toutes les exploitations & $\begin{array}{l}\text { Influence sur le choix de la sous-espèce, uniquement pour les Lolo Rossa } \\
\text { et feuilles de chêne }\end{array}$ \\
\hline
\end{tabular}

quatre structures (qui ne représentent que $15 \%$ à $20 \%$ de la production de leur département), on peut néanmoins considérer qu'elles sont représentatives de la diversité des fonctionnements existant sur le bassin de production.

Le traitement des données a reposé sur des comparaisons entre structures de mise en marché, entre les deux espèces et à l'intérieur de chacune d'elles. Nous avons cherché à mettre en évidence la stratégie collective de la structure de mise en marché. Cependant, on sait que les agriculteurs coordonnent les systèmes de culture à l'échelle de l'exploitation agricole par des processus itératifs et hiérarchisés d'attribution des ressources aux espèces et cultures [11-13] et qu'une action de la structure de mise en marché sur les choix techniques perturbe ce processus [10]. Dans notre étude, les difficultés de mise en ouvre de la stratégie collective, dans les exploitations, ne seront pas analysées de façon exhaustive, mais seulement citées pour expliquer un certain nombre de freins observés dans la planification des plantations.

Nous avons traité les données en deux temps : tout d'abord, nous avons analysé individuellement le cas de la salade et celui de la pêche en recherchant des invariants dans les dispositifs de planification des plantations pour chaque espèce, puis nous avons interprété la variabilité observée entre structures de mise en marché. Dans un deuxième temps, nous avons cherché à comparer les dispositifs des deux espèces en mobilisant des connaissances agronomiques sur les modes d'élaboration du rendement et de la qualité.

\section{Résultats}

\subsection{Le cas de la salade}

L'étude sur la salade a mis en évidence une volonté des structures de mise en marché d'influer sur le choix des sous-espèces et des dates de plantation, mais il a révélé une diversité des degrés d'incitation utilisés d'une structure à l'autre (tableau II).

Chez toutes les structures de mise en marché enquêtées, la mise en place d'un dispositif cherchant à garantir leur approvisionnement avant la plantation des cultures 
a pu être constaté. Ce dispositif et l'embauche d'un technicien datent du début des années 1990, avant même que n'aient pu intervenir les incitations de la nouvelle Organisation commune du marché (OCM) des fruits et légumes. Cette observation a concerné aussi bien les coopératives ( $\mathrm{S} 1$, S5) que les expéditeurs privés (S2, S3, S4). L'objectif commun de ces structures est de mettre en adéquation, dès juin-juillet, à l'échelle du bassin d'approvisionnement, les prévisions de plantations et les prévisions de débouchés pour la campagne à venir (août-mars) ; cela se traduit par des incitations sur le choix des sous-espèces et des dates de plantation. Les différentes actions qui se succèdent dans le temps ont été décrites, pour la structure S1 qui est celle où le dispositif de coordination est le plus complet. On peut considérer ce dispositif comme un modèle général théorique dont dérivent des modèles plus simples caractérisant les autres structures enquêtées.

- En juin, lors d'une réunion de tous les agriculteurs apporteurs, le technicien fait un bilan de la campagne précédente, en indiquant notamment les manques et les excès de salade, décade par décade, sous-espèce par sous-espèce. Il indique également les prévisions de débouchés pour la campagne à venir, pour chaque décade et chaque sousespèce. Ces prévisions proviennent soit de contrats déjà signés (notamment avec les usines de quatrième gamme), soit de discussions informelles entre le commercial et ses clients.

- Chaque producteur établit ensuite un planning de plantation prévisionnel qui tient compte des orientations de l'exploitation (espèces prioritaires, objectifs de production, moyens en superficie, équipement et personnel disponibles sur l'exploitation, etc.) et des informations fournies par le technicien.

- Le technicien rassemble l'ensemble des calendriers de plantation et estime les volumes qui seront produits à l'échelle du bassin d'approvisionnement, sur la base d'hypothèses de durée des cycles et de pourcentage de pertes (variables suivant les sousespèces, les périodes de l'année et entre cultures de plein champ et sous abris). Pour chaque sous-espèce, il définit les périodes d'inadéquation avec le calendrier prévisionnel des débouchés.

- Il propose alors à certains producteurs des adaptations de leur calendrier de plantation pour que la courbe d'offre s'ajuste mieux à la courbe de demande.

La planification des plantations se déroule sur environ 1 mois. Elle porte sur la totalité de la campagne, mais l'attention est portée sur le premier cycle de culture qui va être planté dans le mois qui suit. En effet, pour les cycles suivants, des adaptations peuvent avoir lieu en cours de campagne, sans que des réunions de planification n'aient lieu à nouveau de façon formelle.

Le choix du dispositif de planification présenté s'explique par le positionnement commercial de la structure S1 (tableau I). Elle a passé des contrats avec une usine de salade de quatrième gamme et s'est engagée à fournir certaines sous-espèces peu cultivées dans la région (chicorée de printemps sous abri, jeunes pousses, frisée très fine), à des périodes peu classiques car climatiquement risquées (début et fin de campagne). En effet, les usines de quatrième gamme font fréquemment des mélanges de plusieurs sous-espèces et cherchent à disposer des différentes sous-espèces sur la plus longue période possible pour rentabiliser les équipements. Par le dispositif de coordination mis en place, la structure S1 décrite cherche à s'assurer que les contrats qu'elle a passés, et sur lesquels elle s'est engagée financièrement, seront honorés.

Le dispositif de planification des plantations des autres structures de mise en marché enquêtées est soit similaire (S4), soit plus simple que celui de la structure S1. Ainsi, la structure S2 planifie les plantations, mais cela seulement avec certaines exploitations de son bassin d'approvisionnement : deux exploitations appartenant à la structure (qui fournissent $40 \%$ du volume de la structure) et un groupe d'apporteurs associés au sein d'un Groupement d'intérêt économique (GIE) qui commercialisent la totalité de leur production par S2. La coordination avec les autres exploitations, qui ont des relations commerciales moins régulières, se limite à quelques ajustements à la marge des calendriers de plantation individuels. Cette structure S2 considère que la 
planification de 50-60\% des volumes suffit à garantir ses engagements. La structure S3 intervient sur le choix des dates de plantation, comme le fait la structure S1, pour éviter les creux et pics de production. En revanche, elle ne cherche pas à influer sur le choix des sous-espèces plantées, bien qu'elle produise, comme $\mathrm{S} 1$, des salades à destination de la quatrième gamme. En effet, cette structure S3 possède sa propre usine de quatrième gamme (tableau I) dont le but est avant tout d'écouler la production non commercialisable en frais ; l'usine n'impose donc pas de sous-espèce de salade spécifique, mais s'adapte aux produits fournis par le bassin d'approvisionnement. Une conséquence citée par le technicien est que, dans ce contexte, les coûts de production de la salade de quatrième gamme sont beaucoup plus élevés que lorsqu'il s'agit d'usines indépendantes de la structure de première mise en marché. La structure S5 ne planifie finement que certains produits de diversification pour lesquels les débouchés sont faibles en quantité (bien que très intéressants financièrement) ; la structure cherche à limiter les volumes produits aux seuls débouchés bien identifiés, contrairement à la laitue ou la batavia considérées comme des produits standards.

Par ailleurs, en plus de la planification des plantations, certaines structures mettent également en place un système d'achat collectif de plants : cette action qui est généralement présentée comme un service apporté aux producteurs pour les faire bénéficier de prix plus intéressants est aussi, voire même surtout, une procédure de coordination. En effet, le technicien de la structure, en commandant les plants au pépiniériste à la place du producteur, s'assure que seules les sous-espèces et variétés préconisées seront plantées. Il s'assure également du respect du calendrier de plantation, puisque les plants livrés par le pépiniériste ne peuvent attendre plus de 2 ou 3 jours avant plantation.

\subsection{Le cas de la pêche}

L'étude de la pêche a mis en évidence une tentative des structures de mise en marché pour influencer le choix du matériel végétal sans que le processus ne soit réellement abouti (tableau III).

En pêche, les professionnels ne parlent pas de planification des plantations, mais de politique de plantation. Les quatre structures enquêtées ne cherchent à influer que sur le choix du matériel végétal producteur (greffon), c'est-à-dire les sous-espèces (pêche blanche, pêche jaune, nectarine blanche, nectarine jaune) et les variétés. Le porte-greffe, la densité de plantation et la forme fruitière sont décidés par l'arboriculteur en fonction des seules caractéristiques de son exploitation.

Chaque structure cherche à atteindre ou maintenir un ratio entre sous-espèces à l'échelle de son bassin d'approvisionnement. Ce ratio est variable entre structures et dépend entre autres de leurs orientations commerciales. Par exemple, les structures $\mathrm{P} 1$ et $\mathrm{P} 3$ qui sont très orientées vers l'export recherchent en priorité des pêches ou nectarines à chair jaune (tableau III), les fruits à chair blanche n'ayant un débouché important que sur le marché français ; elles veulent également augmenter le pourcentage de nectarines aux dépends de la pêche, fruit jugé plus difficile à consommer, et donc moins demandé. Cependant, le degré d'incitation pour le choix de la sous-espèce est faible, il s'agit principalement d'une information sur les tendances du marché.

À l'intérieur d'une sous-espèce, les structures de mise en marché enquêtées cherchent à influer sur le choix de la variété. Elles fournissent à leurs apporteurs :

- une liste de variétés recommandées à la plantation,

- une liste de variétés recommandées à l'arrachage.

Ces listes sont élaborées par le technicien de la structure de mise en marché, souvent assisté par une commission constituée de quelques producteurs et du commercial.

Pour élaborer la liste de variétés recommandées à la plantation, le technicien recueille des informations sur les nouvelles variétés proposées par les pépiniéristes, leurs performances agronomiques et la 


\section{Navarrete et al.}

Tableau III.

Dispositifs de planification des plantations de pêchers pour quatre structures de mise en marché, soumises à une enquête dans le sud-est de la France.

\begin{tabular}{|c|c|c|}
\hline Structure & Objectif de la structure & Dispositif \\
\hline \multirow[t]{2}{*}{ P1 } & $\begin{array}{l}\text { Diviser par deux le nombre de variétés } \\
\text { commercialisées }\end{array}$ & $\begin{array}{l}\text { Liste de variétés à planter } \\
\text { (avec trois degrés : besoin de plantation très important, important, moyen) } \\
\text { Commission technique pour élaborer les listes }\end{array}$ \\
\hline & $\begin{array}{l}\text { Atteindre } 60 \% \text { de nectarines } \\
\text { et } 60 \% \text { de chair jaune }\end{array}$ & $\begin{array}{l}\text { Information sur les créneaux de dates à développer } \\
\text { Achat groupé des plants }\end{array}$ \\
\hline \multirow[t]{2}{*}{ P2 } & $\begin{array}{l}\text { Diviser par trois le nombre de variétés } \\
\text { commercialisées }\end{array}$ & Liste de variétés à planter et à arracher \\
\hline & $\begin{array}{l}\text { Maintenir le ratio de } 25 \% \text { dans chaque } \\
\text { sous-espèce }\end{array}$ & \\
\hline \multirow[t]{2}{*}{ P3 } & $\begin{array}{l}\text { Diviser par deux le nombre de variétés } \\
\text { commercialisées }\end{array}$ & Liste de variétés à planter et à arracher \\
\hline & $\begin{array}{l}\text { Atteindre } 65 \% \text { de chair jaune } \\
\text { et } 60 \% \text { de nectarines }\end{array}$ & \\
\hline P4 & $\begin{array}{l}\text { Réduire à } 80 \text { le nombre de variétés } \\
\text { commercialisées }\end{array}$ & $\begin{array}{l}\text { Liste de variétés à planter et à arracher } \\
\text { Commission technique pour élaborer les listes } \\
\text { Information sur les créneaux de dates à réduire (production précoce de juin) }\end{array}$ \\
\hline
\end{tabular}

qualité des fruits. Parmi elles, il effectue un choix limité de nouvelles variétés, en fonction des produits que la structure veut développer, du degré d'adaptation des variétés aux conditions pédoclimatiques locales et des risques agronomiques encourus ; la liste est réactualisée chaque année en fonction des innovations variétales. L'existence de cette liste ne permet pas de piloter les dates de récolte (puisque tous les créneaux de production sont couverts), mais de réduire la diversité variétale dans un créneau de dates donné pour faciliter l'allotement. La deuxième liste regroupe des variétés dont la qualité est jugée inadaptée aux exigences actuelles de la filière ; elle est établie suite aux discussions entre le technicien et les commerciaux.

Le degré d'incitation est très variable d'une structure à l'autre. Généralement, les listes de variétés (arrachage et plantation) n'ont qu'un rôle d'information. Parfois, elles sont accompagnées de grilles de prix, officielles ou officieuses, qui précisent les primes et réfactions appliquées en fonction des variétés par rapport au prix de vente effectif aux clients. C'est le cas pour les structures P1, P3 et P4, par exemple. Dans le cas de $\mathrm{P} 4$, la mesure va jusqu'au refus de commercialiser les variétés jugées obsolètes et préconisées à l'arrachage.

Deux structures (P1 et P4) cherchent en plus à influer sur les dates de récolte. Comme pour la salade, ces créneaux résultent d'une confrontation entre offre et demande à l'échelle du bassin d'approvisionnement, et ils sont définis pour chaque sous-espèce de pêche. L'inventaire des vergers de chaque apporteur (rendu obligatoire par la nouvelle OCM Fruits et Légumes) précise, parcelle par parcelle, la surface, la variété et l'année de plantation. Le technicien somme l'ensemble des inventaires individuels, puis traduit les surfaces par variété en estimations de tonnage et de date de production. Il confronte alors le calendrier de production et le calendrier des débouchés potentiels, fourni par les 


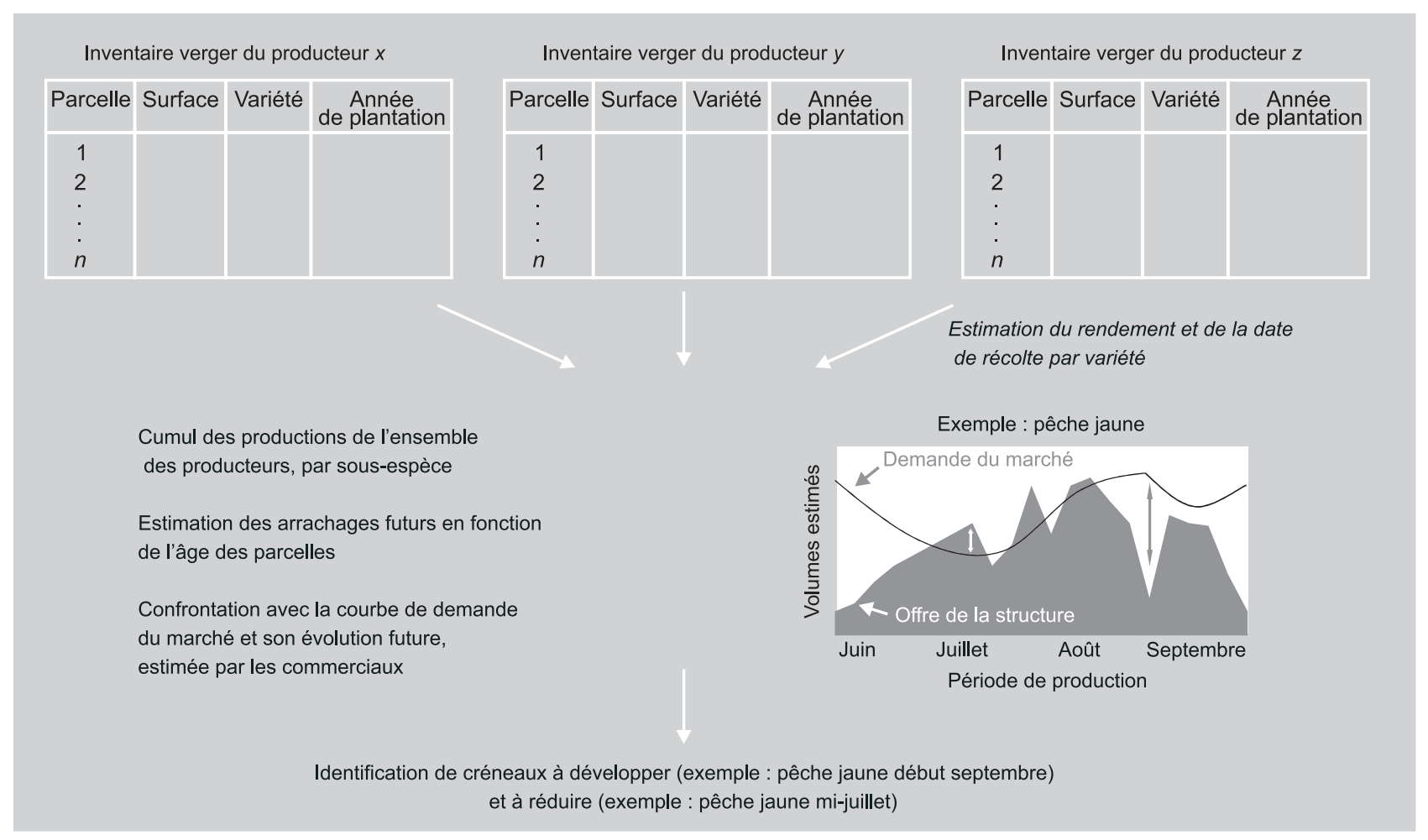

commerciaux, et établit les créneaux de date où la production collective doit être augmentée ou réduite (figure 1). Cependant, dans ces deux structures de première mise en marché, cela ne se traduit pas par des incitations économiques.

\subsection{Les enseignements de la comparaison salade/pêche}

\subsubsection{Un modèle général de planification des plantations...}

L'analyse de la planification des deux espèces salade et pêche montre que les structures de mise en marché ont différents moyens de peser sur les choix techniques des producteurs et en combinent généralement plusieurs : réunion de concertation, discussions bilatérales technicien-agriculteur, listes de variétés positives ou négatives, achat groupé des plants. Le technicien joue un rôle fondamental d'interface entre les producteurs et les commerciaux de la structure de mise en marché. Il dispose d'outils de représentation de la diversité du bassin d'approvisionnement plus ou moins précis Figure 1. (inventaire verger, calendrier de plantation) Définition d'une politique de et de modèles agronomiques (souvent informels) pour faire des estimations de la proDéfinition d'une politique de
plantation par une structure de mise en marché de la pêche. duction à venir.

La planification des plantations est un dispositif de coordination à la fois vertical et horizontal :

- vertical dans le sens où il existe une relation entre la structure et chaque agriculteur,

- horizontal dans le sens où chaque relation verticale est affectée par les relations de la structure avec les autres agriculteurs de son bassin d'approvisionnement. En particulier, c'est le technicien qui négocie les ajustements du calendrier de plantation, en demandant des modifications préférentiellement à certains agriculteurs. De leur réponse dépendent ensuite les demandes faites aux autres. L'évolution du dispositif de coordination au cours des années passées, telle qu'elle est décrite par les techniciens, fait apparaître la planification des plantations comme un processus d'apprentissage progressif, qui cherche à corriger les erreurs 
de l'année passée et à faire évoluer progressivement l'offre en produits, pour s'adapter aux grandes tendances du marché (par exemple, l'élargissement de la période de production, le développement de nouveaux produits de diversification). Lorsque des changements importants des systèmes de culture sont rendus nécessaires par l'apparition rapide d'un nouveau débouché, ils ont généralement lieu dans les exploitations du bassin d'approvisionnement qui ont un statut particulier (soit qu'elles appartiennent à la structure, soit qu'elles lui soient liées par un GIE).

Dans le cas de la pêche, espèce pérenne, une difficulté supplémentaire vient de l'importance de la dimension temporelle dans la gestion du bassin d'approvisionnement. La structure de mise en marché fait un pronostic de la situation à moyen terme, du point de vue de l'offre et de la demande en produits. L'offre en produits au niveau du bassin d'approvisionnement évolue chaque année, mais lentement, en fonction de l'arrachage de vieilles parcelles et de l'entrée en production de parcelles plantées 3 à 4 ans auparavant (le taux de renouvellement est de l'ordre de $10 \%$ des surfaces par an). Les commerciaux cherchent à estimer la demande en produits à moyen terme en s'appuyant sur la demande actuelle et sur de grandes tendances (augmentation des nectarines aux dépends des pêches, déplacement des créneaux de consommation, évolution de la production des autres pays européens, etc.). Mais la gestion en est beaucoup plus délicate que sur salade où l'anticipation ne porte que sur les 2 à 3 mois à venir.

\subsection{2. ...mais des leviers techniques spécifiques à chaque espèce}

L'hypothèse de départ était que les moyens qu'ont les structures pour se coordonner avec leurs apporteurs varient entre espèces suivant les modes d'élaboration de la production. Les résultats montrent que les structures de mise en marché enquêtées cherchent à influer sur le choix de la sousespèce et de la date de plantation en ce qui concerne la production de salade, sur le choix de la sous-espèce et de la variété pour la production de pêche. D'après l'ana- lyse bibliographique présentée précédemment, il apparaît que la sous-espèce en salade et en pêche contraint fortement les caractéristiques du produit et donc les possibilités d'allotement. C'est donc logiquement le levier utilisé par la structure de mise en marché pour maîtriser la variabilité des lots. De même, l'analyse bibliographique a également fait apparaître le poids de la date de semis pour la salade et de la variété pour la pêche dans la définition de la date de récolte. Ce sont effectivement les leviers utilisés par les structures pour piloter la date de récolte, à une nuance près en salade: la majorité des producteurs ne sèment pas, mais achètent les plants de salade à un pépiniériste; la coordination porte sur l'étape de la plantation, la durée entre le semis et la plantation variant peu pour une espèce et un système de production donnés. Les enquêtes confirment donc que les dispositifs de planification des plantations visent à jouer sur l'échelonnement des dates de récolte et les possibilités d'allotement à l'échelle du bassin d'approvisionnement, et qu'ils sont cohérents d'un point de vue agronomique : ils portent sur les actes techniques qui sont les principaux déterminants de ces deux facteurs. Inversement, les structures ne cherchent pas à influer sur les actes techniques qui ne jouent pas ou jouent peu sur ces facteurs, à savoir la densité de plantation, la forme fruitière et le porte-greffe.

L'importance accordée aux variétés de pêche s'explique par la double influence de la variété sur la date de récolte et sur les possibilités d'allotement, d'autant que l'innovation variétale très forte sur cette culture conduit à une multiplicité des variétés. Dans le cas de la salade, la variété ne joue que sur les caractéristiques du produit, le nombre de variétés disponibles reste limité et leurs caractéristiques compliquent peu l'allotement.

\section{Discussion et conclusion}

Les résultats obtenus sur la salade et la pêche ont été comparés aux études antérieures suivant quatre points : l'intérêt de 
la planification des plantations, son degré d'efficacité tant économique qu'agronomique, et enfin les difficultés que rencontrent les agriculteurs dans sa mise en œuvre concrète dans leurs exploitations.

Rappelons que les structures de mise en marché enquêtées cherchent, à travers la planification des plantations, à contrôler à la fois les dates de récolte et la variabilité des lots. La coordination des dates de récolte apparaît d'autant plus nécessaire que le produit est périssable. En effet, lorsque les produits doivent être commercialisés ou transformés très rapidement après récolte, les structures de mise en marché ou de transformation contrôlent les facteurs qui jouent sur la date de récolte : les dates de semis pour les légumes de conserve [2], les dates de plantation pour la salade en frais ou de quatrième gamme. Inversement, lorsque les produits sont stockables, il n'y a pas ou peu de coordination des dates de récolte ; c'est le cas sur céréales [9] et sur la pomme [4]. La pêche, qui peut être conservée pendant une à deux semaines après récolte, se trouve en situation intermédiaire : les structures de mise en marché cherchent à jouer sur le choix des variétés, parce que c'est le principal déterminant des dates de récoltes, mais aussi et surtout parce que la diversité variétale complique l'allotement. Concernant le contrôle de la variabilité des lots, les structures de mise en marché cherchent, au moment de la plantation, essentiellement à jouer sur le choix des sous-espèces et des variétés. La question variétale est cruciale pour la pêche étant donné le nombre de variétés commercialisées et leurs différences qualitatives ; il l'est beaucoup moins pour la salade.

Les risques économiques liés à la planification des plantations par les structures de mise en marché varient fortement suivant qu'il s'agit d'espèces annuelles ou pérennes. Dans le cas de la culture de salades, où chaque cycle dure 2 à 3 mois, les risques pour l'agriculteur sont limités dans le temps ; des ajustements sont possibles en cours de campagne, sur les cycles culturaux suivants. De plus, certaines structures enquêtées proposent des contrats à leurs producteurs avec garantie de prix à certaines périodes climatiquement risquées, voire même une mutua- lisation des risques sur la campagne. On constate que la planification des plantations existe depuis plusieurs années en Roussillon et qu'elle est relativement efficace. Ce n'est pas le cas dans d'autres bassins de production comme en Provence où seule une faible part de la salade est commercialisée dans des structures collectives, ou comme en île-de-France où les circuits courts de commercialisation sont majoritaires : dans ces cas, la gestion de la qualité ne se fait quasiment qu'au niveau de l'exploitation individuelle. Dans le cas de la pêche, une culture étant plantée pour 12 à 15 ans, les risques pour l'agriculteur sont élevés. De plus, la variété conditionne non seulement la date de récolte, mais aussi le potentiel de qualité, et donc les possibilités de valorisation des produits. Or les structures de mise en marché ne garantissent pas un prix de vente aux producteurs, malgré l'existence de grilles de prix officieuses en fonction des variétés. Ce non-partage des risques économiques constitue un frein important au contrôle des dates de récolte par les structures de mise en marché. La question de la coordination entre producteurs et première mise en marché, bien qu'identifiée comme une source de problèmes, n'est encore que faiblement prise en considération par les acteurs de la filière pêche. On retrouve en salade comme en pêche l'idée que, pour que la coordination soit acceptable et acceptée par les agriculteurs, il faut un relatif partage des risques et des bénéfices, ce qui soulève notamment des questions de grilles de prix différenciées.

Les difficultés agronomiques liées à la planification des plantations proviennent des interactions entre techniques et avec le climat dans l'élaboration du rendement et de la qualité. Ainsi, du fait des aléas climatiques, non prévisibles au moment des plantations, une coordination sur les dates de plantation (salade) ou les variétés (pêche) ne garantit pas un bon échelonnement des dates de récolte dans le bassin d'approvisionnement, puisque la durée effective des cycles dépend des conditions climatiques locales ; des chevauchements non prévus des périodes de récoltes peuvent avoir lieu entre parcelles. Pour s'adapter aux aléas climatiques en cours de culture, il existe 
donc des procédures de pilotage et d'ajustement en temps réel, en particulier pour les décisions de récolte ; elles ont été identifiées aussi bien sur les légumes de conserve [2] que sur salade [10] : le technicien s'informe de l'état de maturité des parcelles et organise au mieux les récoltes à l'échelle du bassin d'approvisionnement. C'est aussi pour cette raison que la planification des plantations en salade est réactualisée en cours d'année : un décalage d'une date de récolte sur une parcelle entraîne souvent un décalage de la date de plantation pour le cycle suivant. En revanche, si la planification des plantations est insuffisante pour échelonner les récoltes, elle est l'unique moyen pour anticiper et gérer la proportion relative des différentes sous-espèces de pêche (pêches/nectarines, jaunes/blanches) et de salade (laitue/batavia/frisée, etc.). Mais à l'intérieur d'une sous-espèce, c'est un moyen insuffisant pour contrôler la variabilité des lots, car la qualité des produits ne dépend que partiellement des choix faits à la plantation : en pêche, si le potentiel de qualité est sous contrainte variétale, l'expression de ce potentiel dépend fortement des pratiques culturales (fertilisation, irrigation, éclaircissage, taille en vert, récolte, etc.) [14]. La planification des plantations n'est donc qu'un élément d'un dispositif de coordination plus complet entre les producteurs et la structure de mise en marché, qui permet de contrôler un certain nombre d'éléments clés de l'itinéraire technique. Il inclut la définition de cahiers des charges qui codifient certains éléments d'itinéraires techniques, l'organisation du conseil technique et le pilotage des récoltes en temps réel. Celui-ci consiste à évaluer en cours de culture la qualité des produits des différentes parcelles, pour anticiper le tri à la récolte et la fabrication des lots. Sur céréales, pour prévoir un critère de qualité comme le taux de protéine des grains, des estimations sont réalisées à partir d'indicateurs écophysiologiques du fonctionnement azoté mesuré en cours de culture [15]. En maraîchage, c'est le technicien qui effectue des visites 15 jours avant récolte pour estimer visuellement la qualité des salades [10]

Enfin, la planification des plantations soulève un certain nombre de difficultés dans les exploitations. Bien que n'étant pas l'objet de cet article, elles sont apparues dans les enquêtes comme des freins à la coordination entre producteurs et structures de mise en marché. Ainsi, pour choisir les variétés de pêchers, les agriculteurs donnent la priorité à l'étalement du travail (au moment de la taille des arbres et de la récolte) sur leur exploitation, plutôt qu'au développement des créneaux de commercialisation de la structure de mise en marché [4]. Les producteurs de salade sont confrontés également à des questions d'organisation du travail dans leurs exploitations, qui limitent parfois une gestion plus collective des plantations [10]. Cela renvoie directement à la question de l'articulation des décisions entre niveaux organisationnels différents, qui se pose pour de nombreux systèmes de production [16]. L'analyse de la capacité des agriculteurs à s'adapter dans leurs exploitations à des décisions provenant de niveaux d'organisation plus englobants (bassin d'approvisionnement, bassin versant, zone vulnérable, etc.) devient donc une question cruciale pour les agronomes s'intéressant à ces échelles. En effet, il est clair que les marges de manouvre des agriculteurs varient suivant les exploitations. Sur salade, il pourrait être intéressant d'adapter les procédures de coordination à la diversité des exploitations du bassin d'approvisionnement, en fonction de leurs capacités à fournir tel ou tel produit [10]. D'ores et déjà, on constate que certaines structures de mise en marché de la salade segmentent leur bassin d'approvisionnement, spécialisent certains producteurs sur des cultures risquées (début et fin de campagne, produits demandant de la technicité) ou des cultures demandant des investissements élevés (jeunes pousses). Le Bail, s'appuyant sur des recherches conduites en céréaliculture [15], a montré l'intérêt de concevoir de nouvelles organisations de bassin d'approvisionnement (sélection des agriculteurs, choix de systèmes de culture, modes d'organisation des flux de produits et d'information à l'intérieur du bassin, formalisation d'outils de coordination). La construction d'outils de gestion d'un bassin d'approvisionnement constitue ainsi un champ de recherche nouveau pour l'agronomie. 


\section{Remerciements}

Les auteurs remercient M. Le Bail, F. Papy et F. Bressoud pour leurs conseils au cours de l'étude, S. Bellon et M.C. Léouffre pour la relecture du manuscrit.

\section{Références}

[1] Tordjman S., Déterminisme de la stratégie de culture de la salade en Roussillon. Élaboration d'une grille d'analyse des exploitations, Mém. fin études ing. INAPG, Paris, France, 1997, 64 p.

[2] Capillon A., Valceschini E., La coordination entre exploitations agricoles et entreprises agro-alimentaires. Un exemple dans le secteur des légumes transformés, Études Rech. Syst. Agraires Dév. 31 (1998) 259-275.

[3] Ménard C., L'économie des organisations, La Découverte, Paris, France, 1990.

[4] Rouby A., Les choix de plantation des producteurs de pommes et de pêches dans le Bassin Rhône-Méditerranée: facteurs pris en compte dans les vergers et influence d'opérateurs extérieurs, Mém. fin études ing. INAPG, Paris, France, 2001, 64 p.

[5] Goisque M.J., Calendriers de plantation, salade haute définition. Cahier hors-série Fruits et Légumes, Echo des MIN (1994) 44-47.

[6] Chaux C., Foury C., Productions légumières, tome 2 : légumes feuilles, tiges, fleurs, racines, bulbes, Tech. \& Doc. Lavoisier, Paris, France, 1994.

[7] Bruno J.F., Papy F., Mieux gérer la sole de laitue dans la vallée de la Seine, Cah. Agric. 1 (1992) 95-100.

[8] Hilaire C., Giauque P., Pêche : les variétés et leur conduite, CTIFL, Paris, France, 1994.
[9] Le Bail M., Maîtrise de la qualité des céréales à l'échelle du bassin d'approvisionnement d'une entreprise de collecte-stockage. Approche agronomique, Thèse INAPG, Paris, France, 1997, 237 p.

[10] Tordjman S., La qualité des produits agricoles comme le résultat d'une construction commune à l'ensemble des acteurs d'une filière. Cas de la salade, Rapp. interne, Inra/ Oniflhor, Paris, France, 2002, 200 p.

[11] Papy F., Interdépendance des systèmes de culture dans l'exploitation, in: Malézieux E., Trébuil G., Jaeger M. (Eds.), Modélisation des agro-écosystèmes et aide à la décision, INRA Edition, Paris, France, 2001.

[12] Aubry C., Biarnès A., Maxime F., Papy F., Modélisation de l'organisation technique de la production dans l'exploitation agricole : la constitution de systèmes de culture, Études Rech. Syst. Agraires Dév. 31 (1998) 25-43.

[13] Navarrete M., Maxime F., Bressoud F., Tordjman S., Papy F., Planification des conduites culturales et différenciation des produits dans des exploitations maraîchères, Cah. Agric. 8 (1999) 171-179.

[14] Giauque P., Moras P., Moreau-Rio M.A., Scandella D., Kraeutler E., La pêche. Consommation et itinéraire qualité, CTIFL, Paris, France, 1997.

[15] Le Bail M., Le bassin d'approvisionnement : territoire de la gestion agronomique de la qualité des productions végétales. Les entretiens du Pradel "Agronomes et Territoires ", 12-13 septembre 2002, à paraître dans C.R. Agric. Fr., 2003, 13 p.

[16] Papy F., Agriculture et organisation du territoire par les exploitations agricoles : enjeux, concepts, questions de recherche, C.R. Acad. Agric. Fr. 85 (1999) 233-244. 


\section{La planificación de las plantaciones por las estructuras de primera puesta en mercado en el sector de frutas y hortalizas. Comparación del caso de la lechuga y del melocotón en el sudeste de Francia.}

Resumen - Introducción. Las estructuras de primera puesta en mercado intentan planificar las plantaciones de sus proveedores para facilitar la adecuación, durante las cosechas, entre la oferta de productos y la demanda de sus clientes. El objetivo del artículo consiste en analizar los dispositivos de planificación de las plantaciones en dos especies, la lechuga y el melocotón, y mostrar cómo se tienen en cuenta las limitaciones agronómicas de cada especie y las zonas de venta de las estructuras de puesta en mercado. Método de estudio. Se procedió a encuestar a técnicos de 9 estructuras de puesta en mercado del sureste de Francia, así como a agricultores que abastecen dichas estructuras. Resultados. En el caso de la lechuga, todas las estructuras encuestadas influyen en la elección de las subespecies y fechas de plantación. Eso les permite controlar el escalonamiento de las cosechas y la variabilidad de los lotes de productos, pero con grados variables según las estructuras y sus zonas de venta. En el caso del melocotón, intentan influir a los arboricultores en la elección de las subespecies y variedades, concretamente, esto se refleja fundamentalmente en una reducción del número de variedades comercializadas. Debate y conclusión. El estudio permite comparar la planificación de las plantaciones de lechuga (especie anual) y melocotón (especie perenne): interés, grado de eficacia tanto económico como agronómico, dificultades encontradas por los agricultores en sus explotaciones.

Francia / Provenza Alpes Costa Azul / ensaladas / melocotón / planificación de la explotación / planificación regional / siembra escalonada / elección de especies / mercadeo

To access this journal online: www.edpsciences.org 